\title{
NUMERICAL INVESTIGATION OF MODEL FUSIFORM ANEURYSMS: INFLUENCE OF MAXIMUM DIAMETER TO HEIGHT RATIO
}

\author{
NIMMY THANKOM PHILIP ${ }^{1}$, B. S. V. PATNAIK ${ }^{2}$ AND SUDHIR B. J. ${ }^{3}$ \\ ${ }^{1}$ Department of Applied Mechanics \\ IIT Madras, India, 600036 \\ nimmythankomphilip@gmail.com \\ ${ }^{2}$ Department of Applied Mechanics \\ IIT Madras, India, 600036 \\ bsvp@iitm.ac.in \\ ${ }^{3}$ SCTIMST \\ Trivandrum, India, 695011 \\ bjs@sctimst.ac.in
}

Key words: Hemodynamics, Fluid-Structure Interaction, Abdominal aortic aneurysms.

\begin{abstract}
In the current clinical practice, the rupture risk prediction of fusiform aneurysms is based on maximum diameter. This approach does not account for the size and shape dependent cyclic stresses arising due to fluid-solid interaction (FSI). Previous fluid-structure interaction studies by the authors on model two dimensional fusiform aneurysms (abdominal aortic aneurysm (AAA)) has revealed that the maximum diameter to height ratio $(\mathrm{DHr})$ could possibly be used as a critical parameter, since it can signify the hemodynamic and biomechanical stresses [7]. Hence the present study assesses whether the observations from the shape index based 2D simulations hold good in realistic 3D conditions as well. Based on the preliminary investigations, it is hypothesized, that a combination of $D_{\max }$ and $\mathrm{DHr}$ would be a better indicator of rupture risk.
\end{abstract}

\section{INTRODUCTION}

Aneurysms are localized unhealthy segment of an artery resulting due to the weakening of the blood vessel walls. This would exemplify in the form of a local bulge or an outpouching A fusiform aneurysm is a gradual dilation of the complete circumference of the artery, commonly present in the aorta, in the thoracic region or the abdominal region, near the iliac bifurcation. The latter is commonly referred as an abdominal aortic aneurysm (AAA). In the current clinical practice, surgical treatment of AAA is generally considered necessary when the maximum diameter $\left(D_{\max }\right)$ exceeds 5-6cm [1]. Use of diameter as the primary criterion in the decision to intervene, fails to take into consideration aneurysms that rupture at sizes below operative thresholds. Moreover, diameter is a static measure that does not take into consideration the dynamic interactions between local cyclical forces and the biological state of the arterial wall. Vorp et al., [2] argued that, from a bio-mechanical perspective, knowing 
the wall stress in the lumen, can more accurately predict the rupture risk of AAA, because it is the cyclic stress caused by the pulse wave in conjunction with factors which decrease the strength of the wall, which leads to dilatation and ultimately to rupture [1,2]. So earlier research was targeted towards predicting a rupture potential index (RPI) based on the peak wall stress (PWS) and the tensile strength of the blood vessel, using finite element techniques. These studies inferred that the rupture potential of the aneurysms to be greatly influenced by the geometrical parameters like asymmetry, wall thickness, intra-luminal thrombus (ILT) formation etc $[2,3]$.

It should be pointed out that, the above studies failed to develop a robust co-relation between the magnitude of PWS and RPI, since it ignores the effect of hemodynamic factors like WSS, which is responsible for the cyclic loading of the arterial walls. Presently researchers have shifted their attention on understanding the flow dynamics and features inside the diseased segment, trying to develop a more consistent correlation between WSS parameters and the rupture risk [4,5]. It was observed that the mechanics of the arterial wall and the resulting distribution of wall shear stress parameters are influenced by the individual shape, in addition to the size of the aneurysm. The potential for geometry-based indices to assess rupture risk by describing the derivation of a set of global indices, quantifying size and shape of aneurysms is still a possibility. Martufi et al., [6] have defined certain standard shape indices which could be obtained from any patient-specific MR or CT images. Recent FSI study on 2D model fusiform aneurysms with various shape indices, gave insights on the significance of these indices, in predicting hemodynamics of aneurysm geometries. This study helped us to eliminate out to be the primary turbulence intensity predicting rupture risk
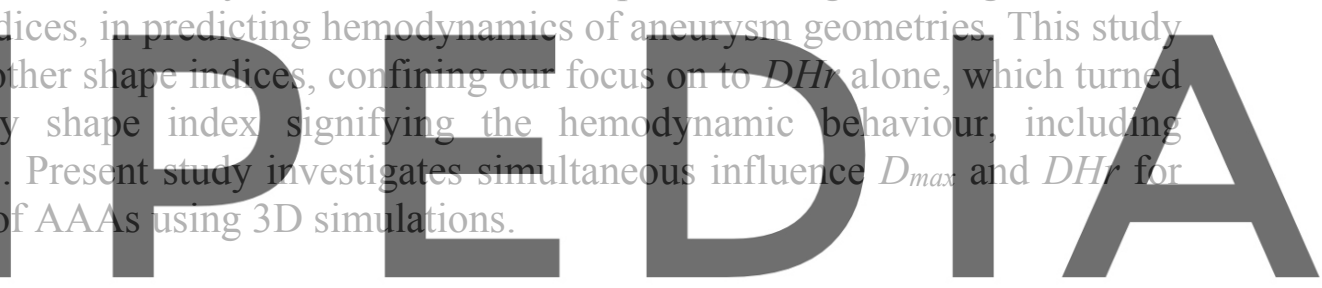

\section{METHODOLOGY}

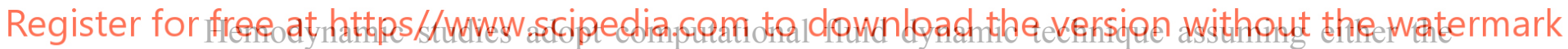
arterial wall to be rigid or numerical simulations with flexible arterial wall accounting for fluid structure interaction. FSI studies are considered to be superior over the rigid wall simulations since it takes into account the strong interactions between flowing blood and the deforming vessel walls. Hence considering its efficiency in accurate modelling of the physics of flow and wall motion, in the present work, FSI based simulations are adopted to study the hemodynamics and bio-mechanics. The coupling of the aneurysmal wall motion and blood flow, at the interface between the fluid and solid, is enabled with the aid of Arbitrary Lagrangian Eulerian (ALE) method, which combines the fluid flow formulated using a Eulerian description and a spatial frame, with wall mechanics formulated using a Lagrangian description and a material (reference) frame. The finite element commercial code COMSOL Multiphysics, incorporating ALE method is chosen for the present study [8].

Even though AAA geometries are patient-specific, for the sake of reducing computational cost and complexity, certain idealizations and assumptions should be made, which could still produce valuable insights into their hemodynamics and biomechanics. Hence present study limits AAA as an idealized axisymmetric diverging and converging segment of an artery proposed by Martufi et al., [6] as given in equation (1). 


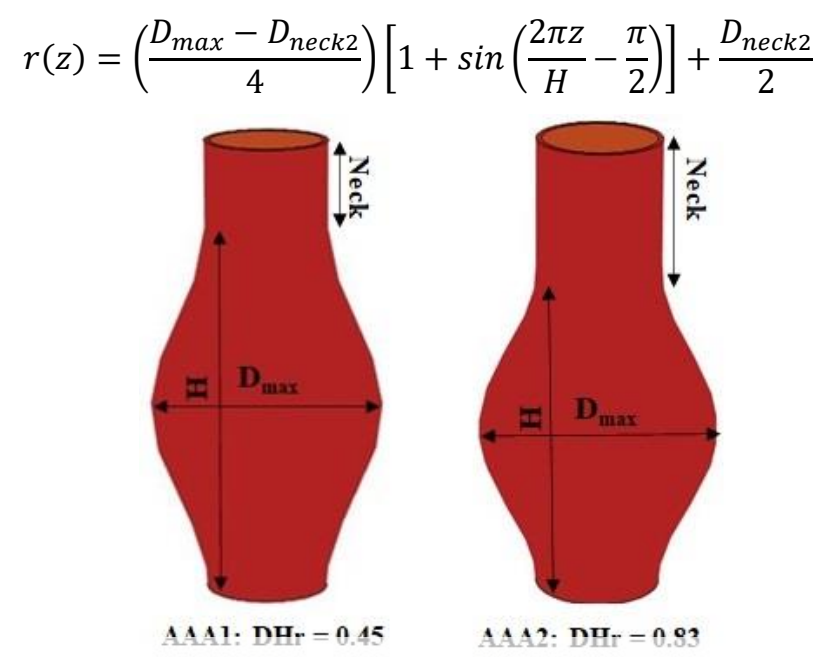

Figure 1: Model geometries used in the present study.

The models used in the present study corresponds to the lowest and the highest value of $\mathrm{DHr}$ reported by Martufi et al., among the nine patient-specific geometries reconstructed by them [6]. The $D_{\max }$ adopted is the clinically accepted critical diameter of $5 \mathrm{~cm}$ as shown in Figure 1. Blood flow is considered to be laminar, in-compressible and Newtonian, the latter owing to blood flows at high shear rates in larger arterios [4,9]. The mass momentur conservation equations and deformed grid. At two conditions viz, the velocity at interface is the forces applied on the interface by blood directions. The set of equations for both solid a

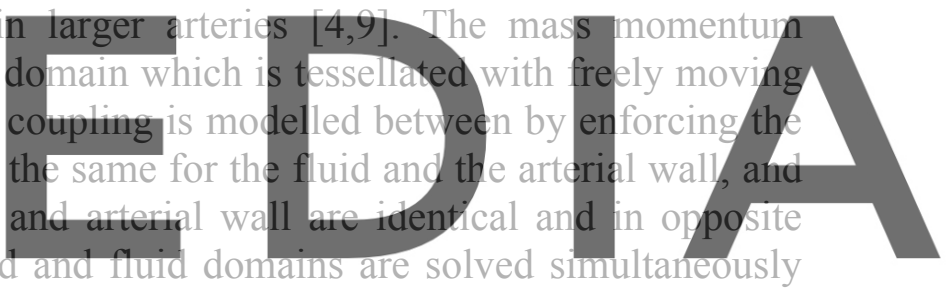
using the fully-coupled approach, with the direct PARDISO solver. The inlet and outlet of the

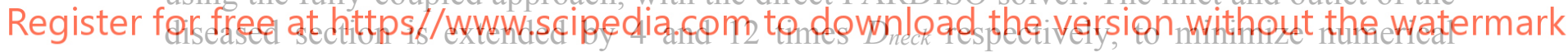
artifacts due to the enforcement of boundary conditions on the inlet and exit sections. Pulsatile velocity and pressure wave forms are enforced as shown in Figure 2 at the inlet and outlet sections respectively.

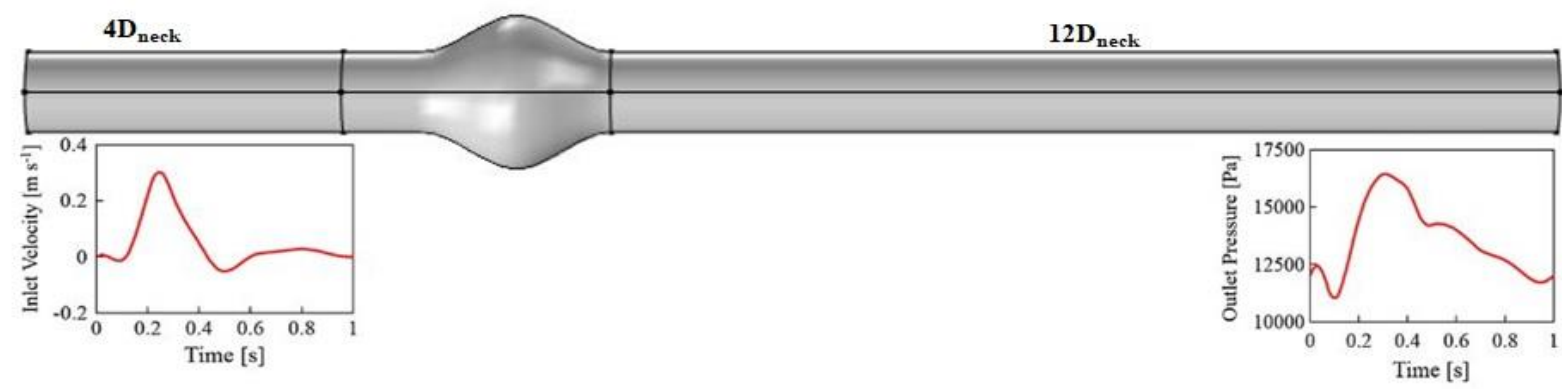

Figure 2: Domain and boundary conditions used in the present study.

Blood is assumed to have a density of $1050 \mathrm{~kg} / \mathrm{m}^{3}$ and a viscosity of 0.0035 Pas and AAA wall is assumed to be, linearly elastic with Young's modulus of $2.7 \mathrm{MPa}$ and Poisson's ratio of 0.45 with a density of $2000 \mathrm{~kg} / \mathrm{m}^{3}$. No-slip boundary condition is applied at the fluid wall, 
with fixed constraints on the side walls of the blood vessel. The outer wall of the blood vessel is set to zero traction. The fluid domain of interest is tessellated with P2/P1 elements and quadratic Lagrange discretization is adopted for the solid domain. Temporal discretization was performed with generalised- $\alpha$ method with time-steps varying from $0.01 \mathrm{~s}$ to $10^{-5} \mathrm{~s}$. Mesh sensitivity studies were conducted on three structured mesh densities namely coarse, fine and finer using the AAA1 geometry. The average flow velocity at the center of the aneurysm section after two cardiac cycles was chosen as the primary variable to establish the grid independence and the relative error between fine and finer mesh was found to be less than $1 \%$. Since the finer mesh had double the mesh resolution in the solid domain, finer mesh is used further in the study. For faster convergence and stability of the FSI simulations, initial two cardiac cycles were simulated with rigid wall assumptions, which served as the initial condition for the flexible wall FSI simulations, executed for the next six cardiac cycles.

\section{Validation of FSI simulation}

An effort was made to validate the methodology adopted for FSI simulation by simulating the classical problem of pressure wave propagation in a flexible tube. Pressure wave is simulated by applying an inlet pressure of $1 \mathrm{KPa}$ at the left end and an outlet pressure of $0 \mathrm{~Pa}$ at the right end of the tube. The tube has a length of $L=0.1 \mathrm{~m}$, a diameter of $D=0.02 \mathrm{~m}$, and a wall thickness of $t=0.002 \mathrm{~m}$. Material properties of the fluid and structure are chosen similar to that of the blood flow in large artery, Young's modulus as $1 \mathrm{MPa}$, Poisson's ratio as 0.3, density of fluid and solid to be same as $10^{3} \mathrm{~kg} / \mathrm{m}^{3}$, and dynamic viscostry of $10^{1} \mathrm{~N} / \mathrm{m}^{2}$. Fixed
constraints were imposed on the side walls of the tube and zero traction was applied on the
outer wall. Computations were carried out on a tetrahedral mesh adopting temporal/spatial
discretization schemes to be same as that is used for the FSI study on andeurysms. Figure 3 (a)
shows the pressure profiles along the tube center line at different time instants, obtaine from the present study compared against the work published by Ha et al. The displacement of the

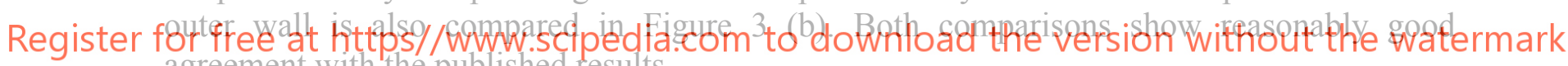
agreement with the published results.

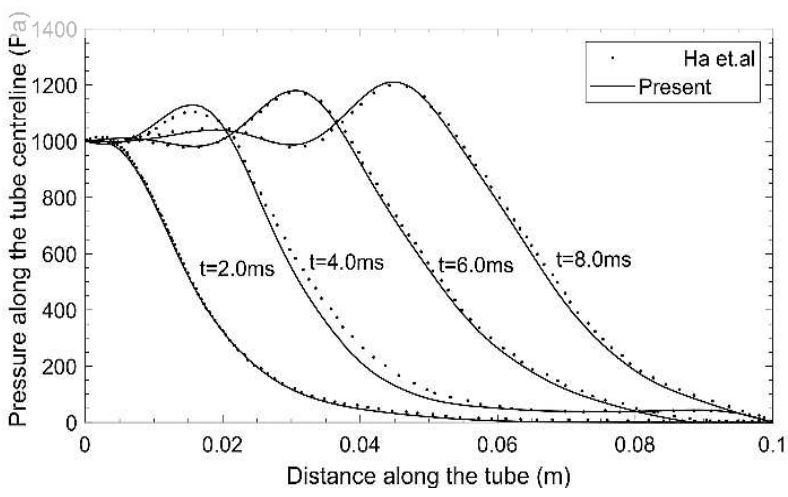

(a)

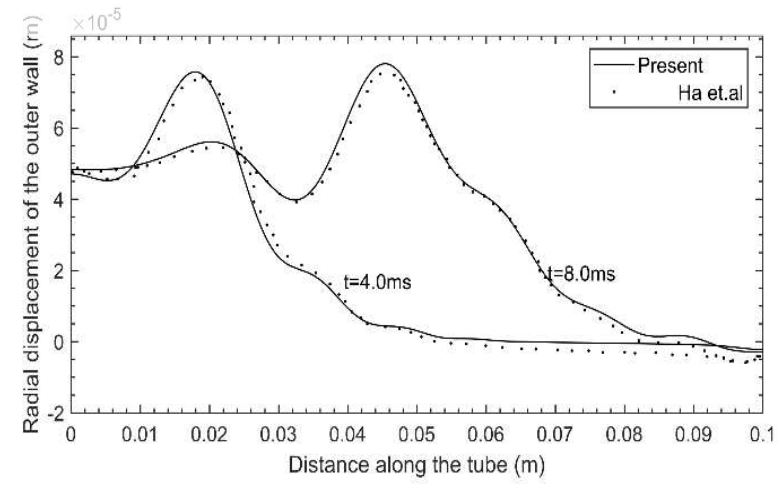

(b)

Figure 3: (a) Pressure wave and (b) outer wall displacement at various time instances compared against Ha et al [10]. 


\section{RESULTS AND DISCUSSIONS}

Fluid structure interaction simulations were carried out to investigate the influence of the expansion ratio $(\mathrm{DHr})$, an index representing the shape of fusiform aneurysms, on the hemodynamic and biomechanics properties of the diseased section. Detailed simulations are performed on two different AAA models to obtain hemodynamic and biomechanical stresses and the influence of $\mathrm{DHr}$ and $D_{\max }$. It is pertinent to investigate, if the hypothesis of the importance of $\mathrm{DHr}$ in 2D simulations [7] is valid for 3D as well and the use of $\mathrm{DHr}$ along with $D_{\max }$ ensures that the fluid stresses (dynamic), its interactions with the wall mechanics, is taken into account, in the decision to intervene.
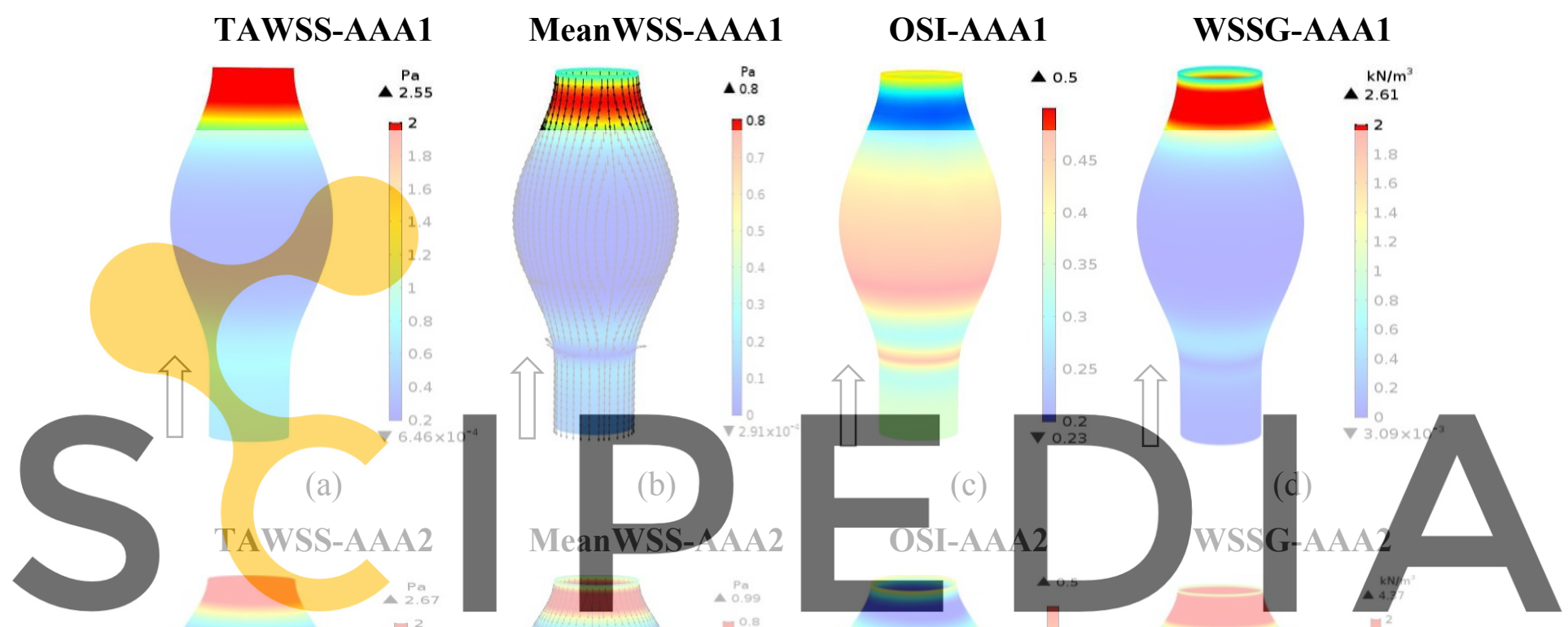

Register for free at https $/ /$ www.scipedia.com ${ }_{0.5}^{6}$ to

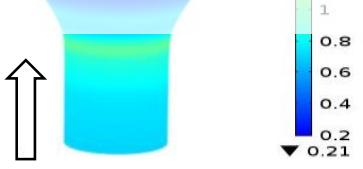

(e)

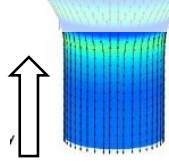

(f)

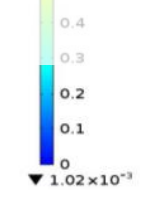

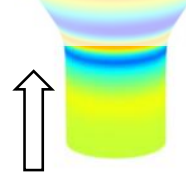

(g)
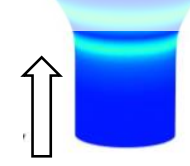

(h)

Figure 4: WSS parameters for AAA1 and AAA2 calculated from the last cardiac cycle.

\subsection{Hemodynamic parameters}

Literature co-relates various WSS parameters such as meanWSS, Time-averaged WSS (TAWSS), oscillatory shear index (OSI), and spatial gradient of WSS (WSSG), with rupture risk/location, thrombus formation, atherosclerosis etc. A detailed explanation on these hemodynamic parameters can be found in Arzani et al [4]. The rupture sites were reported to be near the fluid stagnation regions which have low TAWSS with high WSS gradients, in many of the earlier studies [5,11]. Most researchers, in their studies found larger thrombus growth at the locations with low TAWSS and high OSI [5]. On the contrary, studies on 
smaller aneurysms have reported that thrombus formation is initiated in the regions of high TAWSS and low OSI [4]. Also, atherosclerosis of the aneurysms is reported to be dominant in the regions of high WSSG where wall permeability was higher $[4,11]$. Hence in the present study, to exemplify a hemodynamic perspective, these four WSS parameters are reported along with the flow features.

In general, TAWSS is higher in the distal end of the aneurysm, just after the fusiform shape, and low TAWSS is recorded around the fusiform section. Comparing the TAWSS plots between AAA1 and AAA2 (Figure 4 (a) and 4 (e)), it is clear that the distal end of AAA2 experiences higher TAWSS than AAA1 and TAWSS at the region around $D_{\max }$ is slightly lower for AAA2 than AAA1. Figure 4 (b) and 4 (f) depicts the meanWSS superimposed with its direction. Though the magnitude of meanWSS follows the TAWSS, it changes its direction twice starting from the neck of the diseased section. At the fusiform section, for AAA2 the change in direction takes place at the $D_{\max }$ section whereas for AAA1 the change in direction is observed slightly to the proximal half of the aneurysm. The authors suspect that this change in direction could be very well corelated with the region of low TAWSS, flow stagnation, and ultimately to the rupture location. From these observations, we can conclude that the risk of rupture of AAA2 is slightly higher than AAA1, due to the prevalence of lower TAWSS around the diseased section. Regarding OSI, the distal end of the aneurysm seems to have the low OSI, which is a favorable condition for the initiation of thrombus formation. This is in line with the findings by Rourke et al., from his study on patient-specific aneurysms, that significant thrombus growth is found on the distal part of the (Figure 4 (c) and 4 (g) reveals that the OSI is lower for AAA2, both at the distal and
proximal end, whereas low OSI occurs only at the distal end for AAA1. Hence, based or the
published studies it can be concluded that, aneurysms with high DHr value will be more
susceptible to thrombus initiation and growth. Similarly. The WSSG on the distal end is also higher for AAA2 than AAA1 as shown in Figure 4 (d) and 4 (h), signifying higher wall

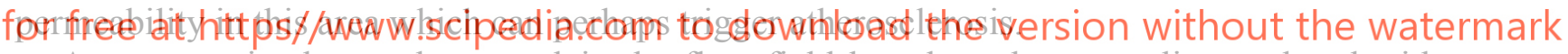

An attempt is also made to explain the flow field based on the streamlines colored with velocity magnitude plot, at uniform time intervals (0.1T) within a cardiac cycle as shown in Figure 5. Forward flow dominates the diseased section only at peak systole, followed by the formation of recirculation zones in early and late diastole. During diastole, regions of flow recirculation is found to originate from walls due to separation and from the central core, due to the expansion of the vessel wall. These recirculation vortices subsequently fill the entire aneurysm cross-section causing flow stagnation (stasis) during late diastole. The temporal acceleration of the flow at early systole induces expansion of the wall which increases the adverse pressure gradient inducing reversed flow in the distal half of the aneurysm. However, peak flow follows early systole and hence it is able to overcome the adverse pressure gradient to generate forward flow till the end of early diastole. This phenomenon is very much explicit in AAA2, which exhibits more cycles of vortex formation and breakdown than AAA1, or in other words the flow field is more dynamics in AAA2 than AAA1. Within a cardiac cycle reversed flow occurred in AAA1 at early and late diastole due to temporal deceleration and just after peak systole due to spatial deceleration, whereas for AAA2 stronger oscillation between forward and reverse flow is observed throughout the cardiac cycle. This explains the oscillatory behavior of meanWSS and lower OSI in the AAA2 model. All these observations 
are consistent with our results from the $2 \mathrm{D}$ simulations and more details on the distinct flow features exhibited by AAA1 and AAA2 can be found in our earlier study [7]. These findings substantiate that the shape index $\mathrm{DHr}$ could be useful to clinicians to assess the risk associated with the progression and rupture of an aneurysm, as all the hemodynamic parameters studied in the literature correlates well with $\mathrm{DHr}$ (expansion ratio).
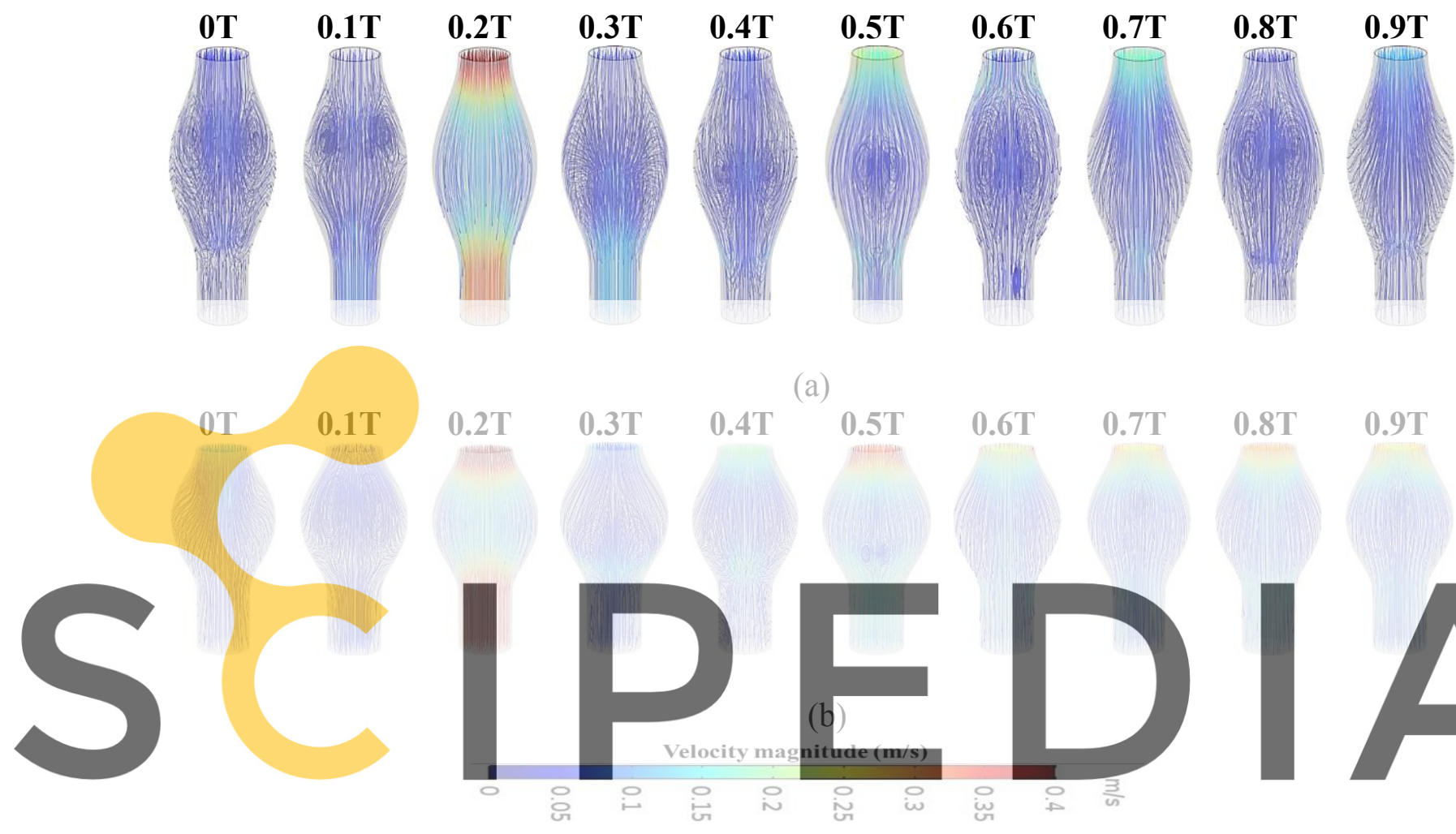

(a)
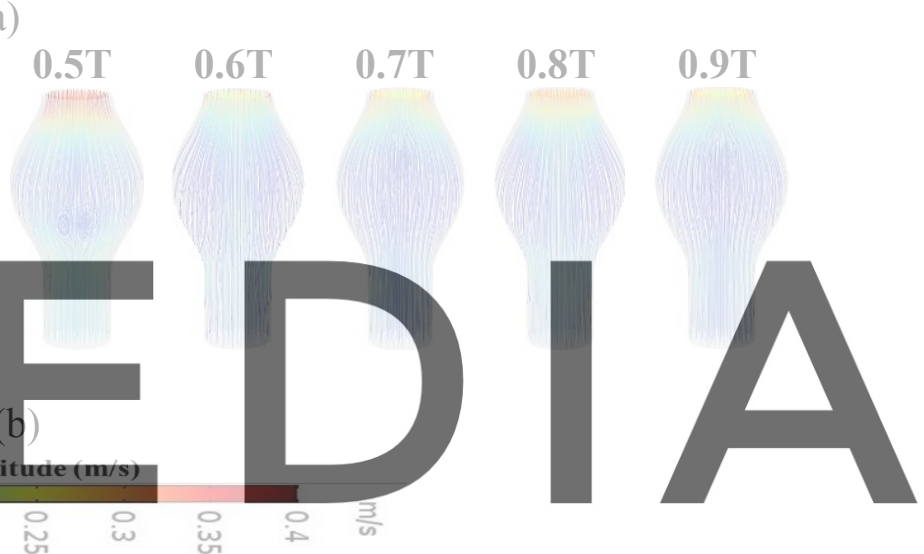

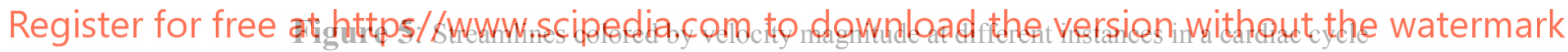

\subsection{Biomechanical Stresses}

In the present study biomechanical stresses are expressed as the vonmises stresses, which is a combination of principal stresses and are used as a failure criterion (in this case aneurysm rupture) of a structure due to internal or external loads. The vonmises stress at peak systole is the peak wall stress (PWS) and the maximum vonmises stress at any instant in a cardiac cycle is the maximum wall stress (MWS). Figure 6 (a) and (b) depicts the PWS and MWS on both inner and outer wall of AAA1 and AAA2. It was observed that irrespective of $\mathrm{DHr}$ the inner wall records slightly higher stresses than the outer wall. Maximum wall stress within the cardiac cycle occurred at the instant of maximum reversed flow for both the aneurysm models. AAA1 shows almost uniform distribution of wall stresses along the fusiform section, whereas in AAA2 a sudden decrease in vonmises stress is observed in and around the $D_{\max }$ section followed by a higher stress in the proximal and distal end of the diseased section. This cyclic oscillations in wall stresses experienced by AAA2 in every cardiac cycle can trigger weakening of the vessel wall due to fatigue. Similarly, the wall expansion and contraction were also observed to be uniform in AAA1 and in AAA2 minimum expansion is seen at the 
$D_{\max }$ section with maximum distortions at the proximal and distal ends. Also, the PWS and MWS is slightly higher for AAA2 than AAA1. Hence it can be concluded that $\mathrm{DHr}$ can be used to differentiate aneurysm geometries biomechanically also.

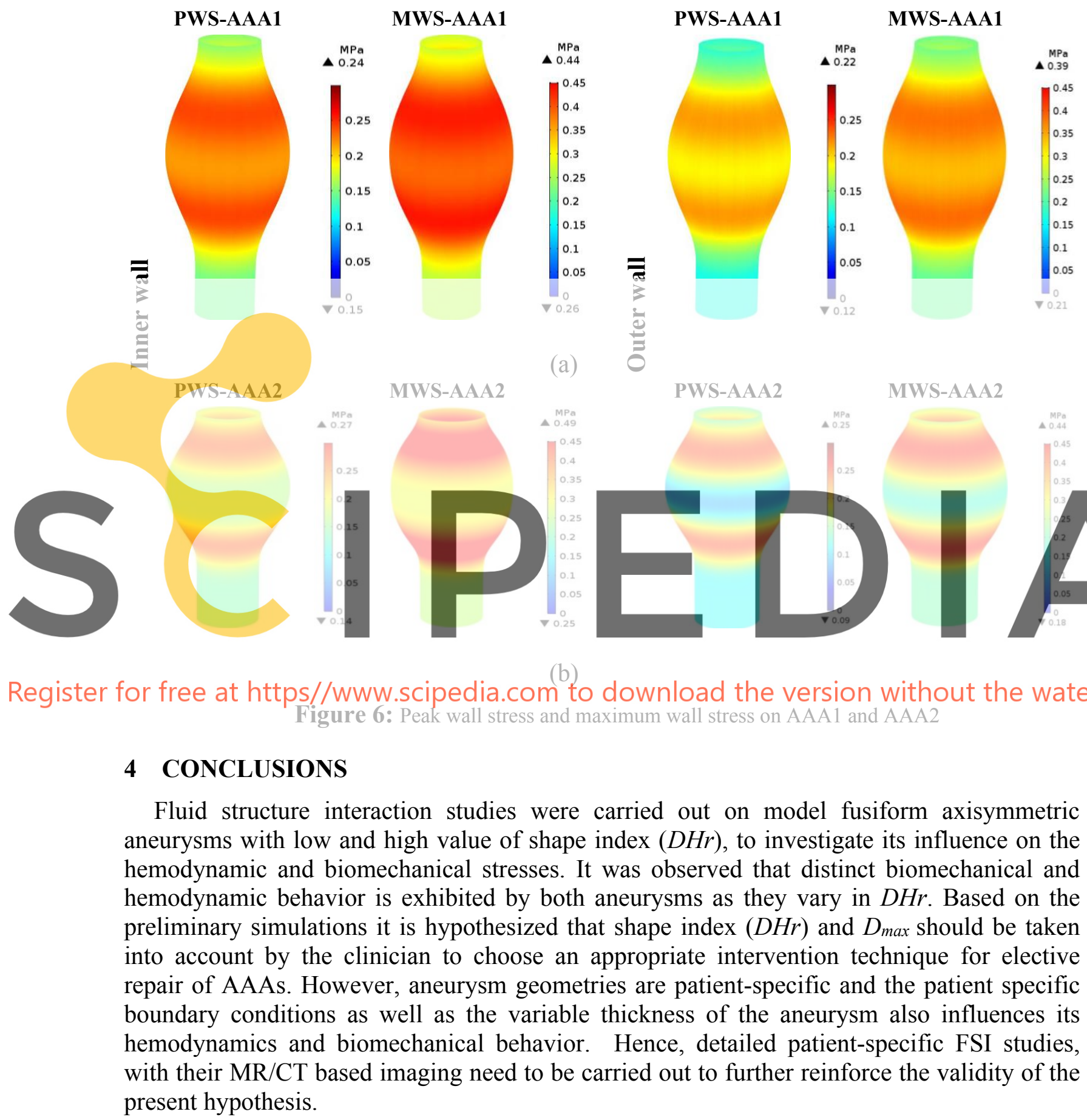




\section{REFERENCES}

[1] Xenos M, Alemu Y, Zamfir D, et al. The effect of angulation in abdominal aortic aneurysms: Fluid-structure interaction simulations of idealized geometries. Medical and Biological Engineering and Computing (2010) 48: 1175-1190.

[2] Vorp D. A, Raghavan M. L, Webster M. W. Mechanical wall stress in abdominal aortic aneurysm: Influence of diameter and asymmetry. Journal of Vascular Surgery (1998) 27(4): 632-639.

[3] Xenos M, Rambhia S. H, Alemu Y, et al. Patient-based abdominal aortic aneurysm rupture risk prediction with fluid structure interaction modeling. Annals of Biomedical Engineering (2010) 38(11): 3323-3337.

[4] Arzani A, Suh G. Y, Dalman R. L, Shadden S. C. A longitudinal comparison of hemodynamics and intraluminal thrombus deposition in abdominal aortic aneurysms. American Journal of Physiology-Heart and Circulatory Physiology (2014) 307(12):H1786-H1795.

[5] Boyd A. J, Kuhn D. C, Lozowy R. J, Kulbisky G. P. Low wall shear stress predominates at sites of abdominal aortic aneurysm rupture. Journal of Vascular Surgery (2016) 63(6): 1613-1619.

[6] Martufi G, Di Martino E. S, Amon C. H, Muluk S. C, Finol E. A. Three-Dimensional Geometrical Characterization of Abdominal Aortic Aneurysms: Image-Based Wall Thickness Distribution. Journal of Biomechanical Engineering (2009) 131: 061015-1-11.

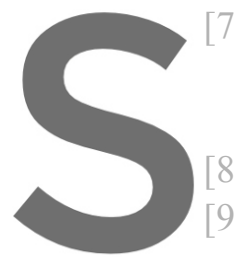
Philip N. T, Patnai abdominal aortic an Biomed. Engng (2020

\section{COMSOL Reference}
Drewe C. Parke

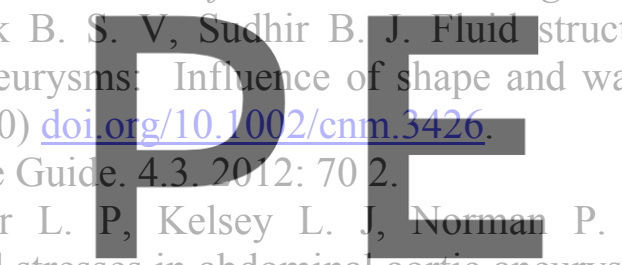

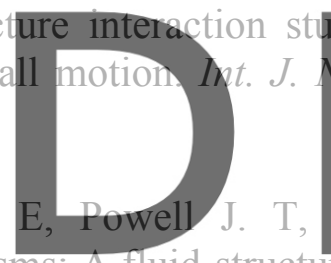

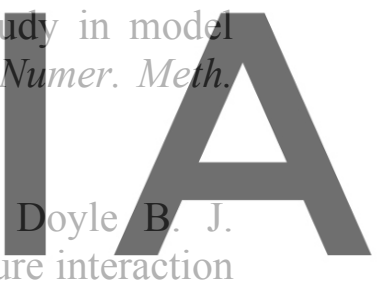

Haemodynamics and stresses in abdominal aortic aneurysms: A fluid-structure interaction study into the effect of proximal neck and iliac bifurcation angle. Journal of Biomechanics

Register for free atshttps./Jyww.scipedia.com to download the version without the watermark

[10] Ha S. T, Ngo L. C, Saeed M, Jeon B. J, Choi H. A comparative study between partitioned and monolithic methods for the probiems with $3 \mathrm{D}$ fiuid-structure interaction of biood vessels. Journal of Mechanical Science and Technology (2017) 30(1):281-287. doi:10.1007/s12206-016-1230-2.15.

[11] Qiu Y, Yuan D, Wen J, Fan Y, Zheng T. Numerical identification of the rupture locations in patient-specific abdominal aortic aneurysms using hemodynamic parameters. Computer Methods in Biomechanics and Biomedical Engineering (2018) 21(1): 1-12.

[12] O’Rourke M. J, McCullough J. P, Kelly S. An investigation of the relationship between hemodynamics and thrombus deposition within patient-specific models of abdominal aortic aneurysm, Proc. Inst. Mech. Eng. Part H J. Eng. Med. (2012) 226:548-564. doi.org/10.1177/0954411912444080. 